\title{
Efecto de gonadotropinas sobre la maduración y desarrollo embrionario de oocitos bovinos cultivados in vitro
}

\author{
Effect of gonadotropins on the maturation and embryo \\ development of bovine oocytes cultured in vitro
}

\author{
Roger Salgado O, ${ }^{1 *}$ M.Sc, Óscar Vergara G, ${ }^{1}$ Ph.D, Leonardo Ramírez $\mathrm{P}^{2}{ }^{2} \mathrm{MVZ}$. \\ ${ }^{1}$ Universidad de Córdoba, Facultad de Medicina Veterinaria y Zootecnia, Departamento \\ de Ciencias Pecuarias. Montería, Colombia. ${ }^{2}$ Ejercicio particular. *Correspondencia: \\ rdsalgado@sinu.unicordoba.edu.co
}

Recibido: Enero 20 de 2009; Aceptado: Agosto 20 de 2009.

\section{RESUMEN}

Objetivo. Evaluar el efecto de las hormonas FSH y LH sobre las tasas de maduración, fertilización y desarrollo embrionario in vitro. Materiales y métodos. Se utilizaron 1309 oocitos de ovarios de matadero, donde el medio base fue TCM-199 suplementado (FSH y LH) y se conformaron cuatro tratamientos al azar: T1: sin hormonas, T2: $50 \mu \mathrm{g} / \mathrm{ml} \mathrm{FSH}+150$ $\mu \mathrm{g} / \mathrm{ml} \mathrm{LH} ;$ T3: $100 \mu \mathrm{g} / \mathrm{ml} \mathrm{FSH} \mathrm{+} 100 \mu \mathrm{g} / \mathrm{ml} \mathrm{LH} \mathrm{y} \mathrm{T4:} 150 \mu \mathrm{g} / \mathrm{ml} \mathrm{FSH} \mathrm{+} 50 \mu \mathrm{g} / \mathrm{ml} \mathrm{LH}$. Resultados. No se presentó efecto $(p>0.05)$ de los tratamientos suplementados con FSH y LH sobre la tasa de maduración; por el contrario, T1 fue diferente $(p<0.05)$ a los demás tratamientos. Se presentó efecto $(p<0.05)$ entre los tratamientos suplementados con FSH y LH sobre las tasas de fertilización. La tasa de divisiones tempranas, no presentaron efecto $(p>0.05)$ cuando el medio de maduración fue suplementado con FSH y LH. Conclusiones. La adición de LH en mayor proporción con respecto a la FSH en el medio de maduración induce una mayor tasa de maduración, fecundación y desarrollo embrionario in vitro a partir de oocitos bovinos recuperados postmortem.

Palabras clave: Oocito, FSH, LH, maduración in vitro

\section{ABSTRACT}

Objective. To evaluate the effect of FSH and LH on the maturation rates, fertilization and embryo development in vitro. Materials and methods. 1309 oocytes were used slaughterhouse ovaries, where the basal medium was TCM-199 plus (FSH and LH) and four treatments were randomized: T1: no hormones, T2: $50 \mathrm{~g} / \mathrm{ml} \mathrm{FSH} \mathrm{+} 150 \mathrm{~g} / \mathrm{ml} \mathrm{LH}$; T3: 100 $\mathrm{ng} / \mathrm{ml} \mathrm{FSH}+100 \mathrm{ng} / \mathrm{ml} \mathrm{LH}$ and T4: $150 \mathrm{~g} / \mathrm{ml} \mathrm{FSH}+50 \mathrm{~g} / \mathrm{ml} \mathrm{LH}$. Results. No effect was found ( $p>0.05)$ from treatments supplemented with FSH and LH on the rate of maturation, by contrast, T1 was different $(p<0.05)$ than the other treatments. Effect was presented 
$(p<0.05)$ among treatments supplemented with FSH and LH on the rates of fertilization. The rate of early divisions, had no effect $(p>0.05)$ when maturation medium was supplemented with FSH and $\mathrm{LH}$. Conclusions. The addition of $\mathrm{LH}$ to a greater extent with respect to FSH in maturation medium induces a higher rate of maturation, fertilization and embryo development in vitro from bovine oocytes recovered postmortem.

Key words: Oocyte, FSH, LH, in vitro maturation

\section{INTRODUCCIÓN}

La producción de embriones bovinos in vitro trae consigo ventajas como el aprovechamiento de las hembras de alto valor genético para la obtención de gran cantidad de descendientes, disminución del intervalo generacional y por ende aceleración del mejoramiento y homogeneidad del componente genético de los hatos ganaderos. Sin embargo, esta técnica tiene limitaciones debido a la baja habilidad de desarrollo al estadio de blastocitos de los oocitos mamíferos madurados in vitro $(1,2)$ ya que muchos de los factores que afectan el proceso de maduración in vivo aun son desconocidos (3).

Los componentes y las condiciones de los medios de cultivo pueden afectar la regulación meiotica de los oocitos mamíferos cultivados in vitro $(4,5)$. Las gonadotropinas hipofisiarias juegan un rol muy importante en la regulación de la maduración de los oocitos (6-8). La hormona folículo estimulante (FSH) actúa en la maduración in vitro a través de las células del cumulus, estimulando la producción de ácido hialurónico y la expansión de estas células, el cual facilita la capacitación espermática, mejorándose así la fertilización y el desarrollo embrionario (9). La hormona luteinizante (LH) estimula la progresión de la meiosis en el oocito del estado de profase a metafase II, en preparación para la fertilización, mediante un decremento del cAMP en el oocito, lo cual es necesario para la transición de profase a metafase (10-13).

Las hormonas LH y FSH, son comúnmente utilizadas en la suplementación de los medios de maduración ya sean independientes o en combinación y en diferentes concentraciones, lo que conlleva a variabilidad entre los resultados de diferentes investigaciones. El objetivo del presente estudio fue evaluar el efecto de las hormonas FSH y LH en diferentes combinaciones y concentraciones en la capacidad de desarrollo de embriones bovinos producidos in vitro.

\section{MATERIALES Y MÉTODOS}

Sitio de estudio. El estudio se realizó en el Laboratorio de Biotecnología de la Reproducción animal "LABRA" de la Universidad de Córdoba, ubicado en el corregimiento de Berástegui, municipio de Ciénaga de Oro, Córdoba, Colombia. La zona se encuentra en un bosque húmedo tropical, altura de $10 \mathrm{msnm}$, temperatura promedio de $32{ }^{\circ} \mathrm{C}$, humedad relativa de $84 \%$ y precipitación anual de $1100 \mathrm{~mm}$ (14).

Recolección de ovarios y oocitos. Los ovarios fueron obtenidos en el matadero municipal de Cereté, Córdoba y transportados al laboratorio en solución salina ( $\mathrm{NaCl} 0.9 \%$ ) suplementada con 100 $\mu \mathrm{g} / \mathrm{ml}$ de estreptomicina a $35^{\circ} \mathrm{C}$. Los oocitos fueron colectados por el método de aspiración directa de los folículos que tenían entre 2 y $5 \mathrm{~mm}$ de diámetro con jeringa de $10 \mathrm{ml}$ y aguja calibre 18 . Una vez aspirados, el contenido de la jeringa fue depositado en una placa de petri de $90 \mathrm{~mm}$ para colectar y clasificar los oocitos grado I y II.

Maduración in vitro. Los oocitos fueron lavados en medio de maduración TCM 199 suplementado con $10 \%$ de SFB, $0.3 \mathrm{mM}$ de piruvato de $\mathrm{Na}, 75 \mu \mathrm{g} / \mathrm{ml}$ de gentamicina y cultivados en gotas de $50 \mu$ en grupos de 10 a 20, cubiertos con aceite mineral, incubados por $24 \mathrm{~h}$ a $38.8^{\circ} \mathrm{C}, 5 \%$ de $\mathrm{CO}_{2} \mathrm{y}$ humedad atmosférica del $96 \%$, distribuidos al azar en cuatro tratamientos: 
$\mathrm{T} 1=$ Sin suplemento de FSH y LH

$\mathrm{T} 2$ = Suplementado con $50 \mu \mathrm{g} / \mathrm{ml}$ de FSH y $150 \mu \mathrm{g} / \mathrm{ml}$ de LH.

T3= Suplementado con $100 \mu \mathrm{g} / \mathrm{ml}$ de FSH y $100 \mu \mathrm{g} / \mathrm{ml}$ de LH.

$\mathrm{T} 4=$ Suplementado con $150 \mu \mathrm{g} / \mathrm{ml}$ de FSH y $50 \mu \mathrm{g} / \mathrm{ml}$ de LH.

Fertilización in vitro. Después de la maduración, los oocitos fueron lavados tres veces en medio de fertilización TAL FERT (TALP Caisson) suplementado con $10 \mu \mathrm{g} / \mathrm{ml}$ de heparina y PHE ( $0.25 \mathrm{mM}$ epinefrina +1 $\mathrm{mM}$ hipótaurina $+3 \mathrm{mM}$ penicilamida). Los espermatozoides se obtuvieron a través del método de Swim-up y se le adiciono a cada gota de fertilización aproximadamente $1.5 \mathrm{x}$ $10^{6}$ esperm $/ \mathrm{ml}$. Los espermatozoides y los oocitos fueron cultivados en gotas de $50 \mu \mathrm{l}$ por $24 \mathrm{~h}$ a $38.8^{\circ} \mathrm{C}, 5 \%$ de $\mathrm{CO}_{2}$ y humedad relativa del $96 \%$.

Cultivo in vitro de embriones. Después de la fertilización, los presuntos zigotos fueron lavados en medio de desarrollo TCM-199 suplementado con $20 \%$ de SFB, $0.3 \mathrm{mM}$ de piruvato y $50 \mu \mathrm{g} / \mathrm{ml}$ de gentamicina. Luego se cultivaron en gotas de $50 \mu \mathrm{l}$ (10 a 20 presuntos cigotos/gota) cubiertas con aceite mineral a $38.8^{\circ} \mathrm{C}, 5 \%$ de $\mathrm{CO}_{2}$, y humedad relativa del $96 \%$. El cultivo se continúo hasta el día 7 post-fertilización.

Evaluación de la maduración y fertilización in vitro. Para la evaluación de la maduración y de la fertilización, a los oocitos que se encontraban en MIV por $24 \mathrm{~h}$ o en FIV por 18 a $24 \mathrm{~h}$, se les retiraron todas las células del cumulus y se procedió a montarlos en una lámina portaobjetos. Luego fueron sumergidos en solución fijadora (una parte de ácido acético/tres partes de metanol) por 24 horas. Posteriormente fueron teñidos con orceina al $2 \%$ en $45 \%$ de ácido acético de 10 a 15 min y fueron examinados en un microscopio de contraste de fase a $100 \mathrm{X}$.

\section{EVALUACIÓN EMBRIONARIA}

Evaluación embrionaria temprana (estado 2-8 células). La evaluación de la división embrionaria temprana se realizó de manera directa el día 3 del cultivo, (el día 0 fue el día de la fecundación), mediante observación con estereoscopio, se evaluaron las tasas de división de 2 a 8 células.

Evaluación embrionaria al estado de mórula-blastocito. La evaluación de la tasa de producción de embriones al estado de mórula (Mo) y blastocitos (B) fue realizada de forma directa en un estereoscopio, observando la cantidad que se encontraban en estos estados avanzados y clasificados en Mo compactas o blastocito, para poder determinar la tasa de producción de embriones para cada tratamiento.

Análisis estadístico. Los resultados correspondientes a la tasa de maduración, fecundación y desarrollo embrionario de los oocitos cultivados in vitro, fueron analizados a través de un análisis de varianza (ANAVA), y se establecieron diferencias significativas por medio de la prueba de Duncan.

\section{RESULTADOS}

Maduración in vitro. No se presentó efecto $(p>0.05)$ en las tasas de maduración in vitro para T2, T3 y T4 respectivamente. Por el contrario, hubo efecto $(p<0.05)$ para T1 con respecto a los demás tratamientos (Tabla 1 ).

Tabla 1. Tasas de maduración in vitro de oocitos bovinos según el tratamiento

\begin{tabular}{lcccc}
\hline \multirow{2}{*}{ Tratamientos } & \multicolumn{2}{c}{$\begin{array}{c}\text { No } \\
\text { oocitos }\end{array}$} & \multicolumn{2}{c}{$\begin{array}{c}\text { Metafase } \\
\text { II (MII) }\end{array}$} \\
\cline { 3 - 6 } & & 96 & 36 & $36.43^{\circ}$ \\
\hline T1: Sin hormonas & $\%$ & $\%$ \\
T2: $150 \mu \mathrm{g} / \mathrm{ml} \mathrm{LH}+50 \mu \mathrm{g} / \mathrm{ml} \mathrm{FSH}$ & 96 & 89 & $92.43^{\circ}$ \\
T3: $100 \mu \mathrm{g} / \mathrm{ml} \mathrm{LH}+100 \mu \mathrm{g} / \mathrm{ml}$ & 96 & 84 & $87.21^{\circ}$ \\
T4: $50 \mu \mathrm{g} / \mathrm{ml} \mathrm{LH}+150 \mu \mathrm{g} / \mathrm{ml} \mathrm{FSH}$ & 96 & 85 & $88.26^{\circ}$ \\
\hline
\end{tabular}

Porcentajes con diferente letra difieren estadísticamente $(p<0.05)$

Fertilización in vitro. Se presentó efecto $(p<0.05)$ en las tasas de fertilización para T2. No hubo efecto ( $p>0.05)$ entre T3 y T4; sin embargo, fueron diferentes a los otros tratamientos. Las tasas de fertilización presentaron $(p<0.05)$ para T1 comparado 
con los tratamientos suplementados con FSH y LH (Tabla 2).

Tabla 2. Tasas de fertilización in vitro de oocitos bovinos seqún el tratamiento.

\begin{tabular}{|c|c|c|c|}
\hline \multirow{2}{*}{ Tratamientos } & \multirow{2}{*}{$\begin{array}{c}\text { No }^{\circ} \\
\text { oocitos }\end{array}$} & \multicolumn{2}{|c|}{ Fecundados } \\
\hline & & No & $\%$ \\
\hline T1: Sin hormonas & 115 & 41 & $35.73^{\circ}$ \\
\hline $\mathrm{T} 2: 150 \mu \mathrm{g} / \mathrm{ml} \mathrm{LH}+50 \mu \mathrm{g} / \mathrm{ml} \mathrm{FSH}$ & 116 & 95 & $81.81^{*}$ \\
\hline T3: $100 \mu \mathrm{g} / \mathrm{ml} \mathrm{LH}+100 \mu \mathrm{g} / \mathrm{ml} \mathrm{FSH}$ & 116 & 82 & $70.63^{\circ}$ \\
\hline $\mathrm{T} 4: 50 \mu \mathrm{g} / \mathrm{ml} \mathrm{LH}+150 \mu \mathrm{g} / \mathrm{ml} \mathrm{FSH}$ & 116 & 75 & $64.63^{\circ}$ \\
\hline
\end{tabular}

Divisiones tempranas (2-8 células). No se presentó efecto $(p>0.05)$ en las tasas de divisiones tempranas (2-8 células) para T2, T3 y T4 respectivamente. Mientras que hubo efecto $(p<0.05)$ para T1 comparado con los demás tratamientos (Tabla 3).

Tabla 3. Tasa de clivaje (2-8 células) in vitro de oocitos bovinos según el tratamiento.

\begin{tabular}{|c|c|c|c|}
\hline \multirow[t]{2}{*}{ Tratamientos } & \multirow[t]{2}{*}{$\begin{array}{c}\text { No } \\
\text { oocitos }\end{array}$} & \multicolumn{2}{|c|}{$\begin{array}{c}\text { Clivaje } \\
\text { (2-8 } \\
\text { células) }\end{array}$} \\
\hline & & No & $\%$ \\
\hline T1: Sin hormonas & 118 & 21 & $19.40^{\circ}$ \\
\hline $\mathrm{T} 2: 150 \mu \mathrm{g} / \mathrm{ml} \mathrm{LH}+50 \mu \mathrm{g} / \mathrm{ml} \mathrm{FSH}$ & 118 & 60 & $53.33^{3}$ \\
\hline T3: $100 \mu \mathrm{g} / \mathrm{ml} \mathrm{LH}+100 \mu \mathrm{g} / \mathrm{ml} \mathrm{FSH}$ & 117 & 56 & $50.91^{\mathrm{s}}$ \\
\hline $\mathrm{T} 4: 50 \mu \mathrm{g} / \mathrm{ml} \mathrm{LH}+150 \mu \mathrm{g} / \mathrm{ml} \mathrm{FSH}$ & 109 & 48 & $48.23^{\circ}$ \\
\hline
\end{tabular}

Desarrollo embrionario al estado de mórula-blastocisto. No Hubo efecto ( $p>0.05)$ entre T2 y T3 sobre las tasas de desarrollo embrionario al estado morulablastocito. Sin embargo T2 fue diferente $(p<0.05)$ a los demás tratamientos; mientras que T3 y T4 fueron similares $(p>0.05)$ pero diferente a T1 (Tabla 4).

Tabla 4. Tasa de producción de embriones in vitro de oocitos bovinos según el tipo de tratamiento.

\begin{tabular}{lcccc}
\hline \multirow{2}{*}{ Tratamientos } & \multirow{2}{*}{$\begin{array}{c}\text { No } \\
\text { oocitos }\end{array}$} & \multicolumn{2}{c}{ Embriones } \\
\cline { 4 - 5 } & No & $\%$ \\
\hline T1: Sin hormonas & 118 & 11 & $10.15^{\circ}$ \\
T2: $150 \mu \mathrm{g} / \mathrm{ml} \mathrm{LH} / 50 \mu \mathrm{g} / \mathrm{ml} \mathrm{FSH}$ & 118 & 41 & $35.83^{\circ}$ \\
T3: $100 \mu \mathrm{g} / \mathrm{ml} \mathrm{LH} / 100 \mu \mathrm{g} / \mathrm{ml} \mathrm{FSH}$ & 117 & 33 & $28.75^{\circ \mathrm{b}}$ \\
T4: $50 \mu \mathrm{g} / \mathrm{ml} \mathrm{LH} / 150 \mu \mathrm{g} / \mathrm{ml} \mathrm{FSH}$ & 109 & 27 & $25.76^{\circ}$ \\
\hline
\end{tabular}

Porcentajes con diferente letra difieren estadísticamente $(p<0.05)$

\section{DISCUSIÓN}

Las tasas de maduración de los tratamientos suplementados con FSH y $\mathrm{LH}$ en las concentraciones y combinaciones utilizadas en el presente estudio estimularon altas tasas de maduración nuclear ( 87 a 92\%). Ha sido demostrado que la LH o FSH sola o en combinación, induce altas tasas de maduración de oocitos bovinos in vitro. Resultados similares han sido comunicados por Ratto et al (15) quienes encontraron a concentraciones de 0.08 UI de h-FSH una tasa de maduración del 93\%; Chohan y Hunter (16) $92 \%$ al utilizar $10 \mu \mathrm{g} / \mathrm{ml}$ de b$\mathrm{FSH}$ y $10 \mu \mathrm{g} / \mathrm{ml}$ de b-LH; Boelhauve et al (17); $84 \%$ y $81.7 \%$ al utilizar $22 \mu \mathrm{g} / \mathrm{ml}$ de FSH ó $8 \mu \mathrm{g} / \mathrm{ml}$ de LH respectivamente.

En la ausencia de gonadotropinas (FSH y LH) solamente el $36.43 \%$ alcanzó la maduración nuclear (Metafase II), lo cual indica la importancia de las gonadotropinas (FSH y LH) para una adecuada maduración in vitro $(10,18,19)$. Este resultado podría ser explicado dado que los oocitos al ser extraídos de los folículos y cultivados en un medio adecuado, inician la maduración nuclear en ausencia de FSH y LH, debido a que son retirados los factores inhibidores de la meiosis presente en el líquido folicular (2023). Resultados similares han sido reportados por Zuelke y Brackett (7), quienes obtuvieron $37.5 \%$ de maduración nuclear en ausencia de hormonas.

En este estudio se demostró que las mayores tasas de fertilización in vitro (81.81\%) fueron alcanzadas cuando el medio de maduración contenía una mayor proporción de LH, lo que demuestra la influencia positiva de esta hormona sobre la tasa de penetración. Esto podría ser explicado por una mayor tasa de maduración nuclear que estaría favoreciendo cambios en la zona pelucida, específicamente a nivel de receptores ZP3, que son los responsables de la unión del espermatozoide al óvulo y participar en el proceso de fertilización (24-26). Resultados similares han sido comunicados por Ratto et al (15) y Chohan y Hunter (16), quienes obtuvieron una tasa de fecundación de $76.9 \%$ y $79.9 \%$ respectivamente. 
Las tasas de fertilización para T3 y T4 fueron similares entre ellos, pero inferiores a T2 indicando el efecto $(p<0.05)$ de la relación hormonal ( $\mathrm{LH}$ y $\mathrm{FSH}$ ), donde a medida que se disminuye la proporción de LH sobre FSH la tasa de fertilización in vitro se ve disminuida (Tabla 2). Resultado similar ha sido comunicado por Zuelke y Brackett (7) quienes obtuvieron $73.5 \%$.

Los medios suplementados con hormonas tienen la capacidad de producir buenas tasas de maduración, fertilización y divisiones tempranas. Los resultados de las tasas de divisiones tempranas en los tratamientos suplementados con hormonas FSH y LH son similares a los comunicados por Chohan y Hunter (16) quienes obtuvieron una tasa de división de $49.4 \%$. No obstante Ratto et al (15) y Choi et al (27) reportaron resultados superiores con tasas de $62.7 \%$ y $66.7 \%$ (en cocultivo de células oviductales y medio condicionado, respectivamente) y $84.3 \%$; mientras que Younis et al (28) reportaron resultados inferiores $37.6 \%$ y $39.6 \%$ para el tratamiento con FSH y LH respectivamente. Por el contrario, una baja tasa de división se presentó cuando los oocitos fueron madurados en ausencia de hormonas $\mathrm{FSH}$ y LH lo que podría inferirse en la falta de adquirir la capacidad de desarrollo embrionario por ausencia del estímulo hormonal (FSH y LH) necesario para la adecuada reanudación de la meiosis $(10,27)$.

La mayor tasa de desarrollo embrionario al estado de mórula-blastocito (35.83\%) fue obtenida a partir de oocitos madurados in vitro con una concentración de hormonas en el medio de maduración de $150 \mu \mathrm{g} / \mathrm{ml}$ de $\mathrm{LH}+50 \mu \mathrm{g} / \mathrm{ml} \mathrm{FSH}$; siendo similar al tratamiento donde el medio de maduración se suplementó hormonalmente con $100 \mu \mathrm{g} /$ $\mathrm{ml}$ de $\mathrm{FSH}$ y $100 \mu \mathrm{g} / \mathrm{ml}$ de $\mathrm{LH}$, pero difiere $(p<0.05)$ con los demás tratamientos. Sin embargo, cuando el medio de maduración fue suplementado con $50 \mu \mathrm{g} / \mathrm{ml} \mathrm{LH}+150$ $\mu \mathrm{g} / \mathrm{ml}$ de $\mathrm{FSH}$ no varió significativamente $(p>0.05)$ del tratamiento suplementado con $100 \mu \mathrm{g} / \mathrm{ml} \mathrm{LH}+100 \mu \mathrm{g} / \mathrm{ml} \mathrm{FSH}$. Por el contrario, una baja tasa $(10.15 \%)$ de embriones al estado de mórula/blastocisto fue observada en el medio de maduración sin la adición de hormonas FSH y LH.

La baja producción de embriones al estado de morula-blastocito (10.15\%) en el tratamiento sin la adición de las hormonas FSH y LH podría ser explicado por fallas en la maduración citoplasmática, lo cual afecta la capacidad de desarrollo de los oocitos fertilizados hasta estos estadios embrionarios (20-22). Resultado similar fue hallado por Zuelke y Brackett (7), quienes obtuvieron una tasa de $7.9 \%$ en el tratamiento sin hormonas durante la maduración.

En conclusión, el presente estudio demuestra la influencia positiva que tienen las gonadotropinas hipofisiarias sobre las tasas maduración, fertilización y desarrollo embrionario in vitro, obteniéndose mejores resultados cuando la proporción de LH fue superior que la FSH en el medio de maduración.

\section{REFERENCIAS}

1. Dielman SJ, Hendriksen PJ, Viuff $D$, Thomsen PD, Hyttel P, Knijn HM, et al. Effect of in vivo prematuration and in vivo final maturation on developmental capacity and quality of pre-implantation Theriogenology 2002; 57:5-20.
2. Rizos D, Ward F, Duffy P, Boland MP, Lonergan P. Consequences of bovine oocyte maturation, fertilization or early embryo development in vitro versus in vivo: implications for blastocyst yeld and blastocyst quality. Mol Reprod Dev 2002; 61:234-248. 
3. Li YH, Jiao LH, Liu RH, Chen XL, Wang $\mathrm{H}$, Wang WH. Localization of angiotensin II in pig ovary and its effects on oocyte maturation in vitro. Theriogenology 2004; 61:447-459.

4. Kito S, Bavister BD. Male pronuclear formation and early embryonic development of hamster oocytes matured in vitro with gonadotrophin, amino acids and cysteamine. J Repro Fertil 1997; 110:35-46.

5. Downs SM, Mastropolo AM. Culture conditions affect meiotic regulation in cumulus cell-enclosed mouse oocyte. Mol Reprod Dev 1997; 46:551-566.

6. Moor RM, Trouson AO. Hormonal and follicular factors affecting maturation of sheep in vitro and their subsequent developmental capacity. J Reprod Fertil 1997; 49:101-109.

7. Zuelke KA, Brackett, BG. Luteinizing hormones enhanced in vitro maturation of bovine oocyte with and without protein supplementation. Biol Reprod 1990; 43:784-787.

8. Saeki K, Hoshi M, Leibfried-Rutledge $\mathrm{ML}$, First NL. In vitro fertilization and development of bovine oocytes matured in serum-free medium. Biol Reprod 1991; 44:256-260.

9. Sirad MA, Desrosier S, Assidi M. In vivo and in vitro effects of FSH on oocyte maturation and developmental competence. Theriogenology 2007; 68(Supl 1): S71-S76.

10. Eppig JJ, Viveiros MM, Bivens CM, De La Fuente R. The Ovary (Second Edition). En: Regulation of mammalian oocyte maturation. Part II. [En linea]. USA: ACADEMIC PRESS; 2003. [fecha de acceso 16 de octubre de 2008]. URL disponible en: http:// www.science-direct.com/science/ book/9780124445628.
11. Mehlmann LM, Kalinowski RR, Ross LF, Parlow AF, Hewlett EL, Jaffe LA. Meiotic resumption in response to luteinizing hormone is independent of a Gi family $\mathrm{G}$ protein or calcium in the mouse oocyte. Dev Biol 2006; 299:345-355.

12. Bilodeau S, Fortier MA, Sirard MA. Effect of adenylate ciclasa stimulation on meiotic resumption and cyclic AMP content on zone-free and cumulusenclosed bovine oocyte in vitro. J Reprod Fertil 1993; 97:5-11.

13. Plancha CE, Albertini DF. Hormonal regulation of meiotic maturation in the hamster oocyte involves a cytoskeleton-mediated process. Biol Reprod 1994; 51:852-864.

14. Instituto geográfico Agustín Codazzi, Córdoba. Características geográficas. Santa Fe de Bogotá: 1995.

15. Ratto $M$, Berland $M$, Wolter $M$. et al. Desarrollo de embriones de bovino obtenidos por fecundación in vitro cultivados con células oviductales o medio condicionado y transferidos a hembras receptoras. Arch Med vet 1999; 31(1):89-96.

16. Chohan KR, Hunter AG. In vitro maturation, fertilization and early cleavage rates of bovine fetal oocytes. Theriogenology 2004; 61:373-380.

17. Boelhauve $M$, Sinowatz F, Wolf E and Paula-Lopes F. Maturation of bovine oocytes in the presence of leptin improves development and reduces apoptosis of in vitro produced blastocysts. Biol Reprod $2005 ; 73: 737-744$.

18. Bevers MM, Dieleman SJ, Van den Hurk $\mathrm{R}$, Izadyar $\mathrm{F}$. Regulation and modulation of oocyte maturation in the bovine. Theriogenology 1997; 47:13-22.

19. Sirard MA. Resumption of meiosis: mechanism involved in meiotic progression and its relation with developmental competence. Theriogenology 2001; 55:1241-1254. 
20. Conti M, Bo Andersen C, Richard F, Mehats C, Chun S, Horner K, et al. Role of cyclic nucleotide signaling in oocyte maturation. Mol Cell Endocrinol 2002; 187:153-159.

21. Sirard MA, Richard F, Mayes M. Controlling meiotic resumption in bovine oocytes: a review. Thenogenology 1996; 49:483-497.

22. Chian RC, Niwa K, Sirard MA. Effects of cumulus cell on male pronuclear formation and subsecuente early development of bovine oocytes in vitro. Theriogenology 1994 ; 41:1499-1508.

23. Luvoni GC, Chigioni S, Perego L, Lodde V, Modina S, Luciano AM. Effect of gonadotropins during in vitro maturation of feline oocyte-cúmulos cell functional coupling and intracellular concentration of glutathione. Anim Reprod Sci 2006; 96:66-78.
24. Bauskin AR, Franken DR, Eberspaecher $U$, Donner P. Characterization of human zona pellucida glycoproteins. Mol Hum Reprod 1999; 5(6):534-540.

25. Bleil JD, Wassarman PM. Sperm-egg interactions in the mouse: sequence of events and induction of the acrosome reaction by a zona pellucida glycoprotein. Dev Biol 1983; 95(2):317-24.

26. Florman HM, Wassarman PM. O-linked oligosaccharides of mouse egg ZP3 account for its sperm receptor activity. Cell 1985; 41(1):313-24.

27. Choi YH, Carnevale EM, Steidel GE, Squires J, Squires EL. Effect of gonadotrophin on bovine oocitos matured in TCM-199. Theriogenology 2001; 56:61-670.

28. Younis AI, Brackett BG, Fayer-Hosken RA. Influence of serum and hormones on bovine oocyte maturation and fertilization in vitro. Gamete Res 1989; 23:189-201. 